\title{
INTERNACIONALIZAÇÃO E
}

DIMENSÕES ORGANIZACIONAIS:

MODELO TEÓRICO-

ANALÍTICO SOB CONTEXTO DA

INTERNACIONALIZAÇÃO

\section{INTERNATIONALIZATION AND ORGANIZATIONAL DIMENSIONS: THEORETICAL-ANALYTICAL MODEL IN THE CONTEXT OF INTERNATIONALIZATION}

\section{D0I: http://dx.doi.org/10.21714/raunp.v10i1.1778}

\section{Nildo Ferreira Cassunde Junior}

Doutor em Administração. Professor da Universidade Federal do Vale do São Francisco. E-mail: nildo.cassunde@univasf.edu.br

\section{Walter Fernando Araújo Moraes}

Universidade Federal de Pernambuco. Professor da Universidade Federal de Pernambuco. E-mail: wfam1950@gmail.com

Envio em: Dezembro de 2017

Aceite em: Janeiro de 2018

\section{ABSTRACT}

Estudos em internacionalização evidenciam a associação com Dimensões Organizacionais, influenciando às competências e habilidades estratégicas da empresa ao longo do tempo. 0 domínio teórico-empírico que envolve os processos de internacionalização pode se apresentar valioso quando é preciso lançar-se em decisões de alocação de recursos e estimular a atividade internacional no país. Neste sentido, este artigo trata de um ensaio-teórico que teve por finalidade apresentar uma proposta de modelo teórico-analítico simulado sob o contexto da internacionalização. A investigação resulta em uma proposição conceitual de um modelo teórico-analítico integrativo que, alicerçado pelas Dimensões Organizacionais, é capaz de descrever a dinâmica existente na internacionalização. A priori, sete dimensões foram consideradas para análise, baseadas em trabalhos seminais e em bibliografia contemporânea prioritariamente internacional alinhando-se, neste particular, ao esforço de pesquisadores brasileiros atuantes na ciência administrativa de aprofundar 0 conhecimento sobre 0 fenômeno que é relativamente recente no meio empresarial, especialmente no que diz respeito a grupos empresariais. A partir da dinâmica dos fatos, na medida em que as estratégias internacionais são adotadas apoiadas em características de internacionalização apropriadas às particularidades de cada organização, as Dimensões Organizacionais passam a emergir ao longo de um recorte temporal. Esta análise retrospectiva revelou a presença destas dimensões ao longo do tempo sob o contexto da internacionalização.

Palavras-chave: Internacionalização. Estratégia. Dimensões Organizacionais. Ensaio Teórico. 


\section{RESUMO}

Internationalization studies show the association with Organizational Dimensions, influencing the company's strategic skills and abilities over time. The theoreticalempirical domain that involves internationalization processes can be valuable when it is necessary to launch decisions on resource allocation and stimulate international activity in the country. In this sense, this article is about a theoretical essay whose purpose was to present a proposal of a theoretical-analytical model simulated under the context of internationalization. The research results in a conceptual proposition of an integrative theoretical-analytical model that, based on Organizational Dimensions, is capable of describing the dynamics of internationalization. A priori, seven dimensions were considered for analysis, based on seminal works and on a contemporary, internationally-based bibliography aligned, in this particular, with the effort of Brazilian researchers in administrative science to deepen knowledge about the phenomenon that is relatively recent in the business world, especially with regard to business groups. From the dynamics of the facts, to the extent that international strategies are adopted based on characteristics of internationalization appropriate to the particularities of each organization, Organizational Dimensions begin to emerge over a temporal cut. This retrospective analysis revealed the presence of these dimensions over time under the context of internationalization.

Keywords: Internationalization. Strategy. Organizational Dimensions. Theoretical Essay. 


\section{INTRODUÇÃO}

A partir de abordagens de natureza econômica ou comportamental, considerando os seminais trabalhos de Vernon (1966, 1979), Johanson e Weindersheim-Paul (1975) e Dunning (1980, 1988), a internacionalização de empresas tem recebido atenção de pesquisadores acadêmicos.

A internacionalização, portanto, tem sido alvo de várias análises e sob diversas perspectivas. Johanson e Vahlne (1977), por exemplo, discutem como um conceito dinâmico, destacando o crescente envolvimento das empresas em operações internacionais. A gestão de riscos nas diferentes fases do processo de internacionalização da empresa, como a ausência de informações precisas sobre o mercado-alvo (JOHANSON; VAHLNE, 2009), pode explicar, segundo Figueira-de-Lemos, Johanson e Vahlne (2011), as alterações no padrão e ritmo do processo de internacionalização. Nesse sentido, Singla e George (2013) revelam que, há três décadas, a literatura internacional tem concentrado esforços para entender como desempenho e internacionalização estão relacionados; no entanto, um número limitado de estudos foca em economias emergentes.

As estratégias de internacionalização revelam aspectos diversos como as influências externas de mercado (PERKS, 2009), mas parecem negligenciar as demais influências da tomada de decisão internacional dos empresários frente aos modos de entrada (POSEBELLO; AMAL; HOELTGEBAUM, 2013), por exemplo. Desse modo, a necessidade de verificar a associação entre as estratégias e os demais fatores influenciadores se torna relevante como forma de diagnosticar os impactos (prioritariamente os negativos) e, a partir disto, trabalhar alternativas que permitam serem compensados por outros fatores.

Neste sentido, estratégias de entrada em mercados internacionais (OSLAND; TAYLOR, ZOU, 2001; MOIZINHO et al, 2014; GARRIDO; LARENTIS, ROSSI, 2006; PERKS, 2009); o comprometimento de recursos (OSLAND; TAYLOR; ZOU, 2001) como os custos de transação (FIANI, 2002) em joint ventures (WILLIAMSON, 1979; 1985; 2007); o fator risco (OSLAND; TAYLOR; ZOU, 2001), entre outros aspectos, podem determinar e favorecer a internacionalização.

Estudos envolvendo estratégia de internacionalização reúnem inúmeras perspectivas e, quando agrupados, revelam que o fenômeno da internacionalização é ditado e marcado por muitas nuances organizacionais. As características da internacionalização, ao longo do tempo, estão englobadas em sete Dimensões Organizacionais, quais sejam: Governança corporativa, Processo de formação da estratégia de internacionalização, Conteúdo das estratégias, Contexto interno, Contexto externo, Riscos percebidos e Empreendedorismo.

Apesar de os estudos de Kovacs (2009) e Leite (2012) também proporem a elaboração de um Framework a partir de determinadas características da internacionalização das empresas, do trabalho de Mota, Machado e Moraes (2014) terem trabalhado fatores que influenciam a internacionalização, e do hexágono organizacional proposto por Moraes et al (2012) procurar analisar as características da internacionalização ao longo do tempo, tais modelos não avançam na análise da associação, ao longo do tempo, entre internacionalização e Dimensões Organizacionais de empresas nem tão pouco abordam o caráter dinâmico que envolver tais relações.

As teorias que dão suporte às características da internacionalização evidenciam que as Dimensões Organizacionais são incorporadas às competências e habilidades estratégicas da empresa, em especial com perspectiva internacional, ao longo do tempo; evidenciando, portanto, o caráter de dinâmico. É importante destacar que estas características podem culminar em um modelo com nuances que revelem sua dinâmica de tal modo que se assume a possibilidade de sofrer transformações ao longo das fases na medida em que se verifica a entrada de novas Dimensões Organizacionais antes não adotadas para o processo de internacionalização.

Partindo-se das considerações teóricas entende-se que o desenvolvimento de estratégias de internacionalização de empresas recebe influência, em diferentes níveis de intensidade, ao longo do tempo, de associações entre as dimensões organizacionais.

Portanto, por entender que a dinâmica entre as Dimensões Organizacionais e a internacionalização, a priori, não apresenta prevalência de causa e efeito, este estudo é orientado pela seguinte questão que se coloca entre a declaração e o campo de investigação, o agronegócio: Como ocorrem, ao longo do tempo, as associações entre as Dimensões Organizacionais, sob o contexto da internacionalização? 


\section{PRESSUPOSTOS TEORICOS}

Estudos envolvendo estratégia de internacionalização reúnem inúmeras perspectivas e, quando agrupados, revelam que o fenômeno da internacionalização é ditado e marcado por muitas nuances organizacionais. Nesse sentido, as características da internacionalização, ao longo do tempo das empresas com atuação no agronegócio, estão englobadas em sete Dimensões Organizacionais. É relevante dizer nesta etapa que, em função do limite máximo de palavras exigidas para o artigo, dada a importância de tais dimensões e o nível atual de discussão na academia sobre elas, optou-se, para este manuscrito, apresentá-las pontual e suscintamente tanto de obras seminais quanto de publicações recentes.

Neste sentido, pesquisas têm sido realizadas com o propósito de compreender o processo de internacionalização das empresas (AMAL et al, 2010; MORAES et al, 2012; PLATCHEK; FLORIANI, 2013; PETRY et al, 2014). Os resultados dos estudos têm mostrado contundentemente que, ao menos, sete Dimensões Organizacionais, alicerçadas nas Teorias de Negócios Internacionais, delineiam as características da internacionalização ao longo do tempo. Tais dimensões são apresentadas condensadamente como se segue. Assim, tem-se que as dimensões organizacionais consideradas para o desenvolvimento da simulação gráfica das associações entre as dimensões organizacionais sob o contexto da internacionalização, quais sejam:

1. Governança corporativa: estruturas organizacionais, sistemas de controle e processos administrativos que permitem a dimensão ser retratada sob a ótica da estrutura de propriedade, da tendência de diversificação, do estilo do executivo e do ponto de vista do risco (JENSEN; MECKING, 1976; WILLIAMSON, 1998; FAMA, 1980; FAMA; JENSEN, 1983; BRICKLEY; COLES; JARRELL, 1997; CORE; HOLTHAUSEN; LARCKER, 1999; LA PORTA et al, 1999; KWEE; VAN DEN BOSCH; VOLBERDA, 2011; FILATOTCHEV; WRIGHT, 2011; KLING; WEITZEL，2011; BARROSO; VILLEGAS; PEREZ-CALERO, 2011; OLIVEIRA; ALBUQUERQUE, PEREIRA，2012; COLLIN et al, 2013; MAIA; VASCONCELOS; DE LUCA, 2013; MOIZINHO el al, 2014; MINETTI; MURRO; ZHU, 2015);
2. Processo de formação da estratégia de internacionalização: o processo de formação (deliberada, emergente ou estratégia hibrida) e mudança estratégica, onde dado o modo de entrada, esta dimensão se propões a buscar respostas para perguntas como: De que maneira se chegou às estratégias? Quem participou destas estratégias? Foi deliberada, emergente? Houve um padrão de formação? Quais os fatores que foram considerados? (CHILD, 1972; JOHANSON; VAHLNE, 1977; MINTZBERG, 1978; REID, 1983; MINTZBERG; WATERS, 1985; PETTIGREW, 1987; OLIVEIRA et al, 2009; STRANGE et al 2009; ERDOĞMUS; BODUR; YILMAZ, 2010; CROWTHER; SEIFI, 2011; DE MACEDO OLIVEIRA, 2011; OLIVEIRA et al, 2012; LIN, 2012; NUWAGABA; NTAYI; NGOMA, 2013; SINGLA; GEORGE, 2013);

3. Conteúdo das estratégias: a dimensão possui diversas óticas e pode ser vista sob a perspectiva da diversificação, alianças, estratégias funcionais e genéricas, o elemento humano e, eventualmente, a renovação da própria estratégia, caracterizando as estratégias de negócio (CHANDLER, 1962; PENROSE, 1979; PORTER, 1986; BARTLETT; GHOSHAL, 1987; MELIN, 1992; HOSKISSON el al, 1993; SULLIVAN, 1994; HOSKISSON et al, 2009; KOVACS, 2009; KWEE; VAN DEN BOSCH; VOLBERDA, 2011; YORKS; NICOLAIDES, 2012; SZCZEPAŃSKI; ŚWIATOWIEC-SZCZEPAŃSKA, 2012; BODNARUK; MASSA; SIMONOV, 2013; LIEN; LI, 2013; ZILLI; VIEIRA; HEINZEN, 2015; VOLONTÉ; GANTENBEIN, 2016);

4. Contexto interno: recursos tangíveis e intangíveis, competência desenvolvidas, evidencia-se a influência no processo de internacionalização a partir dos recursos tangíveis e intangíveis, das competências desenvolvidas e inerentes às empresas (WERNERFELT, 1984; PRAHALAD; HAMEL，1990; PORTER，1990; BARNEY, 1991; PETERAF, 1993; FAHY, 1998; PENG, 2001; FAHY, 2002; OLIVEIRA; MORAES; KOVACS, 2009; BOENE; TONI, 2010; LU et al, 2010; LEITE, 2012; TORRENS; AMAL; TONTINI, 2014; SILVA el al, 2015);

5. Contexto externo: governo, instituições, incentivos, câmbio e barreiras, tudo isso pode ser percebido a partir do ambiente internacional (aspectos associados a políticas governamentais), redução das fronteiras, aspectos de valores e 
culturas, papel da nação- sede, ambiente institucional e relações com o governo (órgão de governo e seus papéis), complexidade e ecleticismo (PIERCY, 1981; DEVIAGGIO; POWELL, 1983; PORTER, 1989; OLIVER, 1991; MEYER; ROWAN, 1992; NARAYANAN; FAHEY, 1999; FARINA，1999; WILD; WILD; HAN, 2006; HENISZ; MANSFIELD, 2006; BURNQUIST et al, 2007; PARRA VILLANUEVA, 2009; JAVALGI et al, 2011; SANTOS; PINHO, 2012; MAGALHAES, RUPOLO; OLIVEIRA, 2014; DE SOUZA AGUIAR; CONSONI; BERNADES, 2014; MAIS; CARVALHO; AMAL, 2014);

6. Riscos percebidos: associam-se ou relacionam-se ao comprometimento de recursos, aos modos de entrada, à velocidade de internacionalizar, ao estilo de direção, à política de incentivos à exportação, à situação política do país-alvo, ao tamanho da empresa, à formação da(s) liderança(s), à percepção e formação dos lideres, experiências anteriores, dentre outros (SHARPE, 1964; MINTZBERG; WATERS, 1985; HARSHMAN; PAIVIO, 1987; LUMPKIN; DESS, 1996; MELIN, 1992; MCCARTHY; LEAVY, 1999; LAM; WHITE, 1999; WELCH; WELCH, 2004; FREITAG FILHO; AMAL, 2008; JOHANSON; VAHLNE, 2009; MELLO; ROCHA; MACULAN, 2009; BUTLER; DOKTOR; LINS, 2010; SCHWEIZER; VAHLNE; JOHANSON, 2010; FERTIS; BAES; LÜTHI, 2012; SZCZEPAŃSKI; ŚWIATOWIEC-SZCZEPAŃSKA，2012; LEITE, 2012; MARTINCORENA et al; 2012; NUWAGABA; NTAYI; NGOMA, 2013; LU; YAN, 2013; LEITE; MORAES, 2014);

7. Empreendedorismo: é caracterizada por aspectos como: oportunismo para criação de valor, propensão em assumir riscos, capacidade de inovar, habilidade em desenvolver recursos competitivos, extenso networking, feeling, liderança, pró-atividade, planejamento, dinamismo e determinação (BIRCH, 1979; VÉRIN, 1982; SHANE; VENKATARAMAN, 2000; JOHANSON; VAHLNE, 2006; AMAL; FREITAG FILHO; MIRANDA, 2008; LINDSTRAND, ERIKSSON, SHARMA, 2009; LANDSTRÖM; LOHRKE, 2010; SCHWEIZER; VAHLNE; JOHANSON, 2010; NASSIF; GHOBRIL; SILVA, 2010; GIBBONS; HENDERSON, 2012; HONÓRIO, 2015; MAGACHO; PRESA; CARNEIRO, 2012; SILVA; CHAGAS; SIQUEIRA, 2012; GE;
WANG, 2013; LEITE; MORAES, 2014; VERGA; SILVA, 2014; MACHADO; NASSIF, 2014; LEITE; DE MORAES; SALAZAR, 2016).

\section{SIMULAÇÃO GRÁFICA DAS ASSOCIAÇÕES ENTRE AS DIMENSÕES ORGANIZACIONAIS SOB O CONTEXTO DA INTERNACIONALIZAÇÃO}

Considerando que:

1. Da revisão de literatura extrai-se que a internacionalização, ao longo do tempo, está associada a seis Dimensões Organizacionais;

2. Outras Dimensões Organizacionais poderão surgir ao longo do tempo e terão sua presença marcada pelo grau relativo de intensidade. É possível que, em um momento seguinte, a "Dimensão A" que outrora fora tida como principal influenciadora passe em algum momento seguinte a ser menos representativa de impacto no contexto organizacional. Ou seja, as Dimensões Organizacionais podem possuir um valor relativo diferenciado ao longo do tempo;

3. É possível que exista associação entre as dimensões (como também, circunstancialmente, pode não haver) e essa associação possuir graus de relacionamentos específicos, ao longo do tempo;

4. O modelo inicial poderá surgir com mais de uma Dimensão a depender, dentre outros fatores, da carga de experiências acumuladas no momento em que antecedeu a internacionalização de suas atividades, ao longo do tempo;

5. Possa existir uma relação dinâmica das / e entre as Dimensões Organizacionais ao longo de toda estratégia de internacionalização. Essa proposição leva em consideração que estudos anteriores desenvolvidos por Moraes et al (2012), Kovacs, Moraes e Oliveira, (2012), Oliveira et al. (2012), ao tratarem da relação tempo no desenvolvimento da estratégia de internacionalização, não revelaram dinâmica entre as dimensões;

6. As abordagens longitudinais, segundo Melin (1992), podem registrar quatro tipos de processos. A exemplo do que se imagina capturar neste estudo, é possível que as diferentes abordagens longitudinais de Melin (1992) sejam encontradas. O autor distingue quatro categorias: 1) o Tipo A ou Série Temporal de Eventos - resume-se ao registro de intercorrências, eventos, pon- 
tuais, como ocorrências criticas provocados por fatos históricos;

2) o Tipo B ou Episódios Relativamente Curtos - resume-se a ocorrências, episódios, com duração de curtas semanas ou anos; 3) o Tipo C ou Longas Épocas ou Temporadas - refere-se ao registro de um longo período de práticas gerencias sob o mesmo fim; e, por fim, 4) o Tipo D ou Histórico Bibliográfico - trata-se de um compendio histórico onde se registra a vida ou um longo período histórico de uma determinada organização, por exemplo.

Nesta investigação, o eixo do tempo adotado segue a proposição de Melin (1992), considerando apenas a Série Temporal de Eventos e os Episódios Relativamente Curtos para ilustração da linha de tempo na simulação gráfica da associação entre as dimensões organizacionais sob o contexto da internacionalização.

A Figura 1 apresenta uma proposta de simulação gráfica das associações, ao longo do tempo, assumindo a relação dinâmica existente entre as Dimensões Organizacionais a partir do contexto da internacionalização. É importante salientar neste momento que não existe um modelo posto, tendo em vista a dinâmica com que podem se apresentar as Dimensões Organizacionais ao longo das fases e a sua relação de associação.

\section{FIGURA 1 - SIMULAÇÃO GRÁFICA DA ASSOCIAÇÃO, AO LONGO DO TEMPO (F) ENTRE AS DIMENSÕES ORGANIZACIONAIS SOB O CONTEXTO DA INTERNACIONALIZAÇÃO}

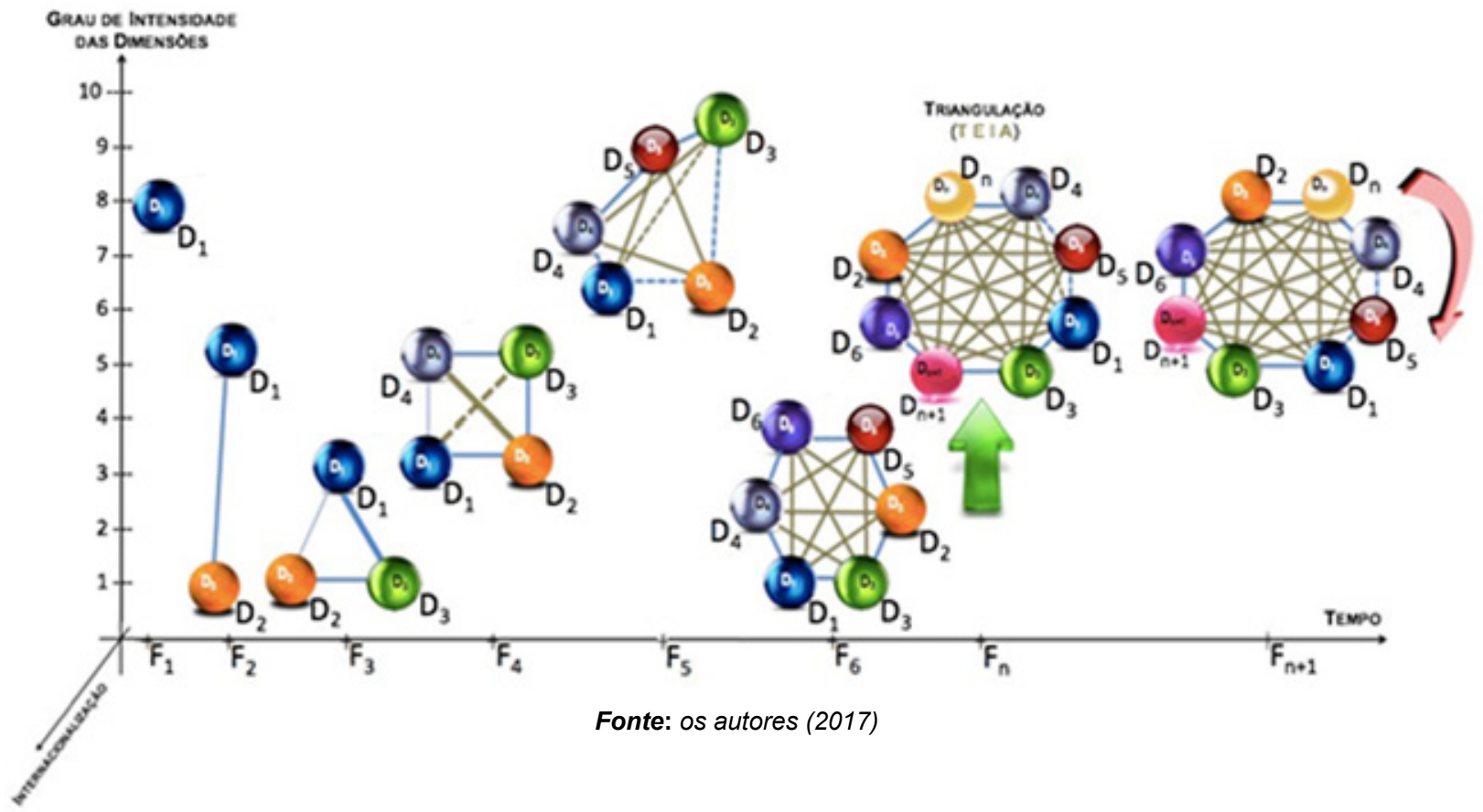

Tal simulação deve ser interpretada a partir dos seguintes apontamentos:

1. A associação da internacionalização com as Dimensões Organizacionais é construída, ao longo do tempo, a partir da intensidade das Dimensões Organizacionais e, por esse motivo, resulta em uma figura plana constituída por vértices e arestas, especialmente quando houver mais de duas Dimensões envolvidas;

2. A escala temporal $F$ (tempo em $F_{1}, F_{2}, \ldots, F_{n}, F_{n+1}$ ) apresenta uma variação a partir de fases ou episódios devidamente identificados na entrevista e da pesquisa documental;

3. O tempo F1 pode iniciar já com mais de uma Dimensão a depender do nível de vivência da empresa no momento em que for iniciada sua trajetória internacional;

4. O grau de inter-relação entre as Dimensões Organizacionais está simbolizado por meio da espessura das linhas que ligam cada uma das Dimensões Organizacionais entre si. Por exemplo: para o momento $F_{3}$ cujo grau de relacionamento entre $D_{1}$ e $\mathrm{D}_{2}$ é menor que $\mathrm{D}_{1}$ e $\mathrm{D}_{3}$, e este menor que $\mathrm{D}_{2}$ e $\mathrm{D}_{3}$; $\mathrm{a}$ linha tracejada, no momento $\mathrm{F}_{4}$, implica inexistên- 
cia de associação; no momento $\mathrm{Fn}$ e $\mathrm{F}_{\mathrm{n}+1}$ é possível registrar que D5 não possui inter-relação direta com D1 e D4, implicando que $\mathrm{D}_{5}$ está relacionado em $1^{\circ}$ grau com as $n$ Dimensões com exceção de $\mathrm{D}_{1}$ e $\mathrm{D}_{4}$;

5. O Modelo formado em cada momento $F$ não, necessariamente, revelará uma figura de dimensões simétricas, a exemplo do Modelo no tempo $\mathrm{F}_{5}$, cuja distribuição a partir do grau de intensidade das Dimensões Organizacionais revelou um Modelo com dimensões não proporcionais entre suas arestas;

6. A triangulação se mostrará presente a partir da presença de três ou mais Dimensões Organizacionais; e a evolução para o Modelo mais complexo não necessariamente é gradual, em que uma dimensão é agregada ao Modelo por vez. É possível que mais de uma Dimensão incorpore ao Modelo em um único episódio, a exemplo do $\mathrm{F}_{6}$ ao $\mathrm{F}_{\mathrm{n}}$;

7. A Intensidade das Dimensões Organizacionais pode mudar entre F's, ou seja, a Dimensão a partir do cenário internacional, pode: (i) mudar de intensidade com o passar do tempo, vide o comportamento da Dimensão 1; (ii) ocupar uma posição relativa diferenciada frente a outras Dimensões entrantes, vide $\mathrm{D}_{1}$ frente a $\mathrm{D}_{2}$ e $\mathrm{D}_{3}$; (iii) quando todas as Dimensões Organizacionais respondem com um grau de Intensidade mais elevada, o Modelo como um todo se desloca para cima, a exemplo da passagem do momento $\mathrm{F}_{6}$ para o $\mathrm{F}_{\mathrm{n}}$ ou para baixo, implicando que as Dimensões Organizacionais como um todo estão fortemente associadas às práticas de internacionalização;

8. Caso, a partir do momento $F_{n}$ para o $F_{n+1}$, não haja surgimento de mais dimensões ao Modelo ainda é possível que: (i) as Dimensões Organizacionais passem a ocupar um posicionamento relativo diferente frente às outras Dimensões; (ii) exista alteração no grau de associação, de relacionamento, entre as Dimensões; (iii) e mantendo-se as posições relativas das Dimensões, o grau de intensidade de cada uma se alterar, é possível que o resultado seja a rotação do poliedro, como representado $\mathrm{F}_{\mathrm{n}+1}$ quando comparado à tn. Esta última hipótese $\mathrm{a}$ priori implicará que a Empresa, ao atingir seu ponto de saturação de integração de Dimensões ao seu Modelo, por já ter alcançado um elevado grau de maturidade em suas práticas organizacionais frente à internacionalização, apenas necessite ajustar seu pool de Dimensões ao novo cenário que se apresente, pois a dinâmica dos cenários e a própria dinâmica da empresa são tão multáveis que essas posições fatalmente se mostrarão variáveis decorrentes de uma deliberada natureza em determinado período e em outra ocasião ser diferente;

9. O terceiro plano, a internacionalização, trata-se justamente do contexto no qual as dimensões estão inseridas. Para fins de ilustração, optou-se em representa-lo ao máximo no plano bidimensional compreendido entre o tempo e o grau de intensidade das dimensões.

As características organizacionais podem variar com os tipos de mercados e a relação de mais intensidade pode alterar o posicionamento do poliedro.

A partir do estado atual em que se evidencia a teoria sobre a internacionalização, as Dimensões Organizacionais parecem emergir de uma soma de percepções onde o resultado parece dar forma a um modelo que, quando assistido do tempo, poderá, facilmente, materializar uma figura composta por ' $n$ ' vértices.

No tocante ao caráter de linearidade, é oportuno esclarecer que a identificação dos fatores capazes de responder o desempenho exportador da empresa avança na medida em que se expandam as variáveis a serem ponderadas (MAIS; CARVALHO; AMAL, 2012). O modelo (até o presente momento listando seis dimensões levantadas) poderá sofrer mutações ao longo da investigação ampliando ou mesmo reduzindo o número de vértices a partir de entendimentos de que as dimensões precisam ser reorganizadas, na medida em que se verifique a presença de mais, ou menos, dimensões.

No tocante a restrições quanto a criação ou ampliação de Dimensões Organizacionais, seguindo as premissas de cada uma das Teorias de Negócios Internacionais - TNI (AMATUCCI; AVRICHIR, 2008; AMAL et al, 2010; MAIS; CARVALHO; AMAL, 2012) e, segundo os estudos realizados (OLIVEIRA; MORAES; KOVACS, 2012; MOTA; MACHADO; MORAES, 2014; OLIVEIRA et al, 2015; LEITE; DE MORAES; SALAZAR, 2016), é importante destacar que a pesquisa visa esgotar os aspectos capazes de responder à formação das estratégias. E desse modo, espera-se que, em determinado momento, defronte-se com o limite onde as nuances ao longo do estudo não mais revelem características suficientemente argumentativas para que estas possam por si constituir uma dimensão e/ou, até mesmo, que esta(s) nova(s) possível(is) dimensão(ões) não seja(m) capaz(es) de justificar ou influenciar o desenvolvimento de estratégias de internacionalização ao longo de sua história.

É importante registrar também que, como a internacionalização é um processo composto por eventu- 
ais descontinuidades em suas operações (RABOCH; AMAL, 2008), o recorte temporal da pesquisa pode não ser capaz de registrar características relevantes para que estas possam compor uma nova dimensão.

Isso posto, é preciso considerar que a simulação originalmente apresentada poderá sofrer transformações e até mutações ao longo de uma investigação ampliando, desse modo, a figura; ou diminuir o número de vértices a partir de entendimentos de que as Dimensões Organizacionais precisam ser reorganizadas. Isso pode resultar em muitas implicações, como: (i) o fato de a empresa carecer de habilidades a partir da falta de percepção de Dimensões Organizacionais e, por esse motivo, não está conseguindo obter o desempenho desejado e, com essa constatação, alterar seu plano de práticas estratégicas com vista a potencializar suas ações empresariais; ou até (ii) verificar que, para se pretender obter desempenhos elevados, é preciso focar em determinadas Dimensões Organizacionais que a própria literatura ainda não havia revelado.

A partir da leitura da teoria, as características passariam por um processo de mutação das habilidades da empresa a partir das Dimensões Organizacionais, e algumas Dimensões Organizacionais podem deixar de ter importância em determinado momento, já que, com a apropriação de outras, a empresa passaria a deixar à margem a dimensão $n$ para valorizar a dimensão $n+1$. A partir de uma elasticidade dos dados no processo, passa-se a incorporar todas as variáveis a partir do tempo; algumas podem deixar de ser as mais relevantes, sem deixarem de existir, pois a teia de relações, aparentemente, não permite que uma dimensão deixe de influenciar o processo mesmo que, essa em determinada circunstância, não surja em primeiro plano na triangulação (o que resultará em uma teia).

A pressuposição é que ela, provavelmente, não perderá conhecimentos. Assim, a empresa ficaria mais robusta e mais sólida em suas dimensões, nas suas decisões estratégicas internacionais, possuindo mais poder deliberativo. É possível entender, portanto, que as Dimensões Organizacionais existentes seriam incorporadas às características da internacionalização a partir da empresa.

Argumento de inter-relação parcial entre as Dimensões Organizacionais subsidia a leitura do modelo para mais um outro entendimento: de que a interação se dê diretamente entre as Dimensões Organizacionais, e quanto mais triangulação existir mais ainda se sustenta a afirmativa de que as dimensões precisem se fazer presentes mesmo que não configurem como ativas em primeiro plano para com as práticas organizacionais de internacionalização. A princípio, sustentadas as características de cada Dimensão, a inter-relação secundária presente no Modelo é sim uma associação que per si já é capaz de manter presente determinada Dimensão que, eventualmente, se mostre menos importante na Fase em estudo.

\section{CONSIDERAÇÕES FINAIS}

O conteúdo das estratégias, enquanto dimensão organizacional, apresentou características diversas, destacando-se: alianças estratégicas com instituições públicas; inovações tecnológicas em especial no desenvolvimento de variedades; transferência de conhecimento gerencial, por meio experiência pretérita ou mesmo por troca de experiências com outras empresas; e estabelecimento de acordos comerciais para expansão de oportunidades no mercado internacional.

Em termos do contexto externo, as empresas são submetidas à padrão elevado de procedimentos de natureza sanitária o que acarreta a elevação dos custos de operação e eventuais procedimentos únicos para vencê-los. Demanda-se também das empresas brasileiras certa astúcia no proceder com alguns intermediários. Significativos gargalos de inadimplências tiveram que ser vencidos com a adoção de iniciativas creditícias próprias. A própria seleção dos mercados apresenta-se repleto de nuances. Políticas governamentais são consideradas de afeição e de aborrecimento, em um processo concomitante, sendo o câmbio a variável mais fundamental quando a questão está associada a internacionalização das operações empresariais.

O risco percebido é predominantemente associado aos fatores exógenos, em especial o fator climático e a política governamental do câmbio. Considerações igualmente são realizadas em relação à questão comercial, inclusive os referentes às denominadas janelas de oportunidades, intencionadas pelos demais concorrentes internacionais. A dinâmica do risco percebido nos negócios desafia o dirigente de forma diuturna, fazendo com que se busque, de forma incessante, alternativas que garantam a sobrevivência do negócio.

O processo de formação da estratégia de internacionalização é ditado pelo aspecto comercial, onde este revela-se fator essencial para o progresso desta dimensão. Os achados evidenciam que o ritmo de in- 
ternacionalização e o escopo da dimensão do processo de formação das estratégias de internacionalização são marcados por características idiossincráticas de gestão. A necessidade de superação dos desafios tem marcado o modo como as estratégias de internacionalização precisam ser construídas de modo a projetar a empresa no mercado internacional. Neste sentido, as relações interpessoais surgem como um plus e auxiliam as empresas a suplantarem as experiências fracassadas, tendo em vista que o jogo de interesses se esforça para manter, sob domínio de poucos, às informações quanto aos potenciais canais comerciais internacionais. Cenário este que a venda em consignado é tido como uma forma ineficaz de alavancar os ganhos.

Os recursos tangíveis e intangíveis, e capacidades próprias estão presentes no contexto interno. Nesta dimensão, a marca, a tecnologia adotada e o know-how gerencial são tidos como fatores preponderantes e determinantes para a trajetória de sucesso das empresas pesquisadas. Escolhido como dimensão para alavancar a inserção de mercado, o contexto interno instrumentaliza as empresas para operar concomitantemente múltiplas ações, levando-as a desempenhar um papel tanto focado no sistema operacional, como tático quanto estratégico.

A governança corporativa apresenta-se sob a forma que suas orientações têm sido historicamente importantes para perpetuar tanto a cultura institucional quanto promover a manutenção das práticas gerenciais e renovação estratégica, assim como têm se revelado uma extraordinária forma de cunhar a habilidade de gerenciar conflitos dentro das hierarquias. A partir do reconhecimento em quanto importante esta dimensão pode ser dentro das empresas, a política de recompensas tem evoluído e vem ajustando-se no sentido de instituir uma equação de prêmios compatível com o reconhecimento que o alto escalão tem diante do papel do funcionário com a atividade fim da empresa.

Em se tratando do empreendedorismo enquanto dimensão organizacional, este se caracteriza por aspectos como: dinamismo e determinação, oportunismo para criação de valor, propensão em assumir riscos, capacidade de inovar e de planejar, habilidade em desenvolver recursos competitivos, extenso networking, feeling, liderança e pró-atividade. Os exitosos projetos de agronegócio do Vale são marcados pelo pioneirismo da dimensão empreendedora que, munida de suas características organizacionais, é responsável em criar projetos sustentados no binômio: recursos competitivos e oportunidades internacionais. O arrojado empreendedorismo tem surtido generosos resultados, tendo em vista que as experiências têm seguido no sentido de multiplicar habilidades comerciais entendendo que é possível especializar-se ao mesmo tempo que se busca diversificar sua carteira de produtos e mercados. Neste sentido, o fator empreendedorismo tem se mostrado essencial para consolidar as oportunidades em projetos virtuosos.

É possível entender que a empresa ao buscar internacionalizar suas atividades ainda não possui habilidades e domínio pleno de suas competências. E assim que tais fatores tão logo sejam absorvidos via um intenso processo de experiências, transformar-se-ão em novos diferenciais competitivos. Neste sentido, o polígono, que na primeira fase poderá surgir com mais de uma dimensão decorrente, dentre outros fatores, da carga de vivência acumulada no momento em que antecedeu a internacionalização de suas atividades, devido a dinâmica de forças pré-existente, indubitavelmente, com o passar do tempo, incorporará novas dimensões a partir do momento que a empresa busque sair da zona de conforto ou até deseje elevar a eficiência do seu custo de oportunidades.

O resultado esperado é que, ao atingir um determinado nível de satisfação entre as dimensões, o polígono, agora completo pelas sete dimensões, passe a girar e/ou até mudar de forma revelando deste modo os ajustes que a empresa precisou passar a fim superar determinados desafios ou até ajustar-se a determinadas condições. É importante registrar que, na busca em se contabilizar ganhos, a relação entre as dimensões organizacionais, eventualmente revelarão forças antagônicas, onde uma relação negativa de associação precisa ser antevista com o propósito de se evitar que determinadas dimensões, quando individualmente analisadas, revelem um jogo contrário de interesses a ponto de anular, neutralizar, ou até prejudicar os planos empresariais com vista a auferir o máximo de divisas possível do empreendimento.

Diante do que foi apresentado, é possível afirmar que este ensaio teórico contribui com um novo significado para o tema internacionalização. Superada a inércia do processo de internacionalização, a simulação expõe o processo decisório pode culminar em ajustes de suas respectivas dimensões organizacionais ao contexto internacional em que a empresa se propôs em atuar. Estas dimensões organizacionais possuem uma dinâmica própria tanto de participação na trajetória de internacionalização das empresas quanto de associação entre elas. E ainda merecem ser 
avaliadas a fim de se identificarem as fragilidades e potencialidades encontradas em cada fase.

Em suma, a associação entre as dimensões identificadas em cada Fase, a partir de distintos graus de intensidade, espelham o sucesso, a estagnação ou fracasso empresarial e merecem ser estudadas com atenção, tendo em vista que a sustentabilidade de seus projetos se faz por meio de uma complexa teia de inter-relações entre as dimensões aqui reveladas. $\mathrm{O}$ exitoso processo de gestão, como um todo, é erguido e se estabelece a partir da habilidade em conciliar o planejar, o organizar, o liderar-executar e o controlar, e, portanto, deve munir-se do máximo de ferramentas a fim de tornar, no particular desta tese, o processo de internacionalização o mais exitoso possível. Neste sentido, a proposta de um modelo Teórico- Analítico simulado sob o contexto da internacionalização tem por desígnio instrumentalizar o processo decisório por meio de nuances contidas nas características das dimensões organizacionais de cada empresa, em especial daquelas com atuação no agronegócio, instrumentalizando os tomadores de decisão a agir inspirado em um modelo com polígonos tanto construídos por fatos pretéritos como o por metas futuras.

Importante destacar que a proposta de um modelo Teórico-Analítico simulado sob o contexto da in- ternacionalização visa instrumentalizar estudos para que se faça possível desenvolver propostas de frameworks teórico-dinâmicos para cada estudo de caso que venha a existir. Onde, espera-se que surja para cada estudo empírico um framework exclusivo tendo em vista a gama de idiossincrasias que perpassa cada empresa.

Dentre as sugestões, propõe-se desenvolver um trabalho quantitativo que possa mensurar os gaps entre o esperado e o realizado entre as Dimensões de cada Fase; e um trabalho qualitativo a fim de que se possa avaliar o comportamento das dimensões, estudar o grau de intensidade e a dinâmica vivenciada ao longo do tempo entre as dimensões; a este método, sugere-se que se faça uma investigação via estudo de multicasos para que se faça uma análise cruzada dos achados.

Propõe-se que se desenvolva um estudo que identifique as zonas de conflito de interesse entre as Dimensões, objetivando reduzir os ruídos e tornar a "máquina empresarial" convergindo esforços em prol do mesmo objetivo. $\mathrm{O}$ fato é que onde estejam ocorrendo relações com forças antagônicas, tipo "cabo-de-guerra", ou conflitos de agência os quais costumam funcionar como "freio de mão" na linha do desenvolvimento de planos estratégicos deveriam canalizar para fortalecer ações em busca de novos ou mais ganhos.

\section{REFERÊNCIAS}

AMAL, M. ; FREITAG FILHO, A. R.; MIRANDA, C. S. M. Algumas evidências sobre o papel das redes de relacionamento e empreendedorismo na internacionalização das pequenas e médias empresas. Faces (FACE/ FUMEC), v. 7, p. 11-25, 2008.

.; RABOCH,H.;AWUAH, G.B.;ANDERSSON,S. Theinternationalization of Multinational Companies (MNCs): an intra-sector comparison among firms from developing and developed countries. ENCONTRO DA ASSOCIAÇÃO NACIONAL DE PÓS-GRADUAÇÃO E PESQUISA EM ADMINISTRAÇÃO (EnANPAD), 2010, Rio de Janeiro. XXXIV EnAnpad 2010, 2010.

AMATUCCI, M.; AVRICHIR, I. . Teorias de Negócios Internacionais e a entrada de multinacionais no Brasil de 1850 A 2007. Revista Brasileira de Gestão de Negócios (São Paulo. Impresso), v. 10, p. 234- 248, 2008.

BARNEY, J. B. Firm resources and sustained competitive advantage. Journal of Management, v. 17, n. 1, p. $99-120,1991$.

BARROSO, C.; VILLEGAS, M. M.; PEREZ-CALERO, L. Board influence on a firm's internationalization. New Answers and Questions Arising from Corporate Governance Research. Corporate Governance: An International Review, July, v.19, n.4, p.351, 2011. 
BARTLETT, C. A.; GHOSHAL, S. Managing across Borders: New Strategic Requirements. Sloan Management Review, v. 28, n. 4, p. 7-17, Summer 1987.

BIRCH, D. G. W. The Job Generation Process. MIT Program on Neighborhood and Regional Change, v. 302, 1979. Disponível em: <http://ssrn.com/abstract=1510007>. Acesso em: 20.ago.2015.

BODNARUK, A.; MASSA, M.; SIMONOV, A. Alliances and corporate governance. Journal of Financial Economics, v.107, p. 671-693, March, 2013.

BOENE, D. M.; TONI, D. Alavancando recursos para a internacionalização de micro, pequenas e médias empresas. In: OLIVEIRA JUNIOR, M. M.. (Org.). Multinacionais Brasileiras. Porto Alegre: Artmed/ Bookman, 2010.

BRICKLEY, J. A.; COLES, J. L.; JARRELL, G Leadership structure: separating the CEO and chairman of the board. Journal of Corporate Finance, v. 3, 1997.

BURNQUIST, H. L.; SOUZA, M. J. P.; BACCHI, M. R. P. ; FARIA, R. N. Análise de Evidências sobre a Importância de Barreiras Técnicas à Exportação de Empresas Brasileiras. Economia Aplicada (Impresso), v. 11, p. 209-229, 2007.

BUTLER, J. E.; DOKTOR, R.; LINS, F. A. Linking international entrepreneurship to uncertainty, opportunity discovery, and cognition. Journal of International Entrepreneurship, v. 8, n. 2, p. 121- 134, 2010.

CHANDLER, A. D. Strategy and structure: Chapters in the history of the industrial enterprise. Cambridge, MA: MIT Press, 1962.

CHILD, J. Organizational Structure, Environment and Performance: The Role of Strategic Choice. Sociology, v. 6, p. 1-21, 1972.

COLLIN, S.-O. Y.; SMITH, E.; UMANS, T.; BROBERG, P.; TAGESSON, T. Mechanisms of corporate governance going international; Testing its performance effects in the Swedish economy, 2004. Baltic Journal of Management, v.8, n.1, p.79-101, 2013.

CROWTHER, D.; SEIFI, S. Corporate Governance and International Business. Ventus. 2011. Disponível em: $\quad<$ http://idl.isead.edu.es:8080/jspui/bitstream/123456789/1784/1/9788776817374.pdf.> Acessado em: 31.02 .2014

DE MACEDO OLIVEIRA, H. Internationalization of Brazilian companies: developing managers as a factor essential for the business strategy. Future Studies Research Journal: Trends and Strategy, v.3, n.1, p.111-29, 2011.

DE SOUZA AGUIAR, H.; CONSONI, F. L.; BERNADES, R. C. Estratégia de internacionalização conduzida: um estudo em redes de franquias brasileiras. RECADM, v.13, n.1, p.114-131, 2014.

DEVIAGGIO, P. J.; POWELL, W. W. The iron cage revisited: institutional isomorphism and collective rationality in organizational fields. American Sociological Review, v. 48, p. 147- 160, 1983.

DUNNING, J. H. The Ecletic Paradigm of International Production: a Restatement and Possible Extensions. Journal of International Business Studies, v. 19, p. 1-31, 1988.

. Toward an eclectic theory of internationalp roduction: some empirical tests. Journal of International

Business Studies, v. 11, n.1, p. 9-31, 1980. 
ERDOĞMUŞ, İ. E.; BODUR, M.; YILMAZ, C. International strategies of emerging market firms; Standardization in brand management revisited. European Journal of Marketing, v.44, n.9/10, p.1410-1436, 2010.

FAHY, J. Resources and global competitive advantage: a study of the automotive components industry in Ireland. Irish Marketing Review, v.10, n.2, 1998.

FAMA, E. F. Agency problems and the theory of the firm. The Journal of Political Economy, v.88, n.2, p. 288-307, 1980.

339, jun. 1983

.; JENSEN, M. Agency problems and residual claims. Journal of Law and Economics, v.26, p. 327-

FARINA, E. M. M. Q. Competitividade e Coordenação de Sistemas Agroindustriais: um ensaio conceitual. Gestão e Produção, v.6, n.3, p. 147-161, dez. 1999.

FERTIS, A.; BAES, M.; LÜTHI, H-J. Robust risk management. European Journal of Operational Research, v.222, n.3, p.663-672, 2012.

FIANI, R. Teoria dos Custos de Transação In: KUPFER, D.; HASENCLEVER, L. (Orgs.) Economia Industrial: fundamentos teóricos e práticas no Brasil. Campus: Rio de Janeiro, 2002.

FIGUEIRA-DE-LEMOS, F.; JOHANSON, J.; VADLNE, J. Risk Management in the internationalization process of the firm: A note on the Uppsala model. Journal of world business (Print), v. 46, n. 2, p. 143-153, 2011.

FILATOTCHEV, I.; WRIGHT, M. Agency Perspectives on Corporate Governance of Multinational Enterprises. (Report). Journal of Management Studies, March, v.48, p.471, 2011.

GARRIDO, I. L.; LARENTIS, F. ; ROSSI, C. A. V. . Orientação para o mercado externo, estratégias de entrada em mercados internacionais e performance internacional: proposição para um modelo conceitual. Base (UNISINOS), São Leopoldo, v. 3, n.1, p. 63-73, 2006.

GE, G.; WANG, H. The impact of network relationships on internationalization process: An empirical study of Chinese private enterprises. Asia Pacific Journal of Management, v.30, n.4, p.1169-1189, 2013.

GIBBONS, R.; HENDERSON, R. Relational contracts and organizational capabilities. Organization Science, v. 23 , n. 5 , p. 1350-1364, 2012.

HARSHMAN, R. A.; PAIVIO, A. Paradoxical sex differences in self-reported imagery. Canadian Journal of Psychology, v.41, p. 303-316, 1987.

HENISZ, W.; MANSFIELD, E. Votes and Vetoes: The Political Determinants of Commercial Openness. International Studies Quarterly, New Jersey, v. 50, n. 1, p. 189-211, Mar. 2006.

HONÓRIO, L. C. Fatores de Estímulo da Internacionalização de Empresas Mineiras de Manufaturados. Revista Economia \& Gestão, v. 15, n. 39, p. 23-44, 2015.

HOSKISSON, R. E.; HITT, M. A.; IRELAND, R D.; HARRISON, J. S. Estratégia Competitiva. São Paulo: Cengage Learning, 2009.

.; JOHNSON, R. A.; MOESEL, D. D. Construct validity of an objective (entropy) categorical measure of diversification strategy. Strategic Management Journal, v.14, n.3, p. 215-235, 1993. 
JAVALGI, R. G.; DELIGONUL, S.; DIXIT, A.; CAVUSGIL, S. T. International Market Reentry: A Review and Research Framework. International Business Review, v.20, n.4, p.377-393, 2011.

JENSEN, M.; MECKLING, W. Theory of the firm: managerial behavior, agency costs and ownership structure. Journal of Financial Economics, v. 3, n. 4, p. 305-360, 1976.

JOHANSON, J; VALNE, J.-E. Commitment and opportunity development in the internationalization process model. Management International Review, v.46, n.2, p. 165-178, 2006.

. Internationalization process of firm - model of knowledge development and increasing foreign market commitments. Journal of International Business Studies, v.8, n.1, p. 23-32, 1977.

. The Uppsala internationalization process model revisited: From liability of foreignness to liability of outsidership. Journal of International Business Studies, v.40, p.1411- 1431, 2009.

JOHANSON, L.; WEINDERSHEIM-PAUL, F. The internationalization of the firm - Four Swedish cases. The Journal of Management Studies, p. 305-322, 1975.

KLING, G.; WEITZEL, U. The internationalization of Chinese companies: Firm characteristics, industry effects and corporate governance. Research in International Business and Finance, v.25, n.3, p.357-372, 2011.

KOVACS, E. P. O processo de internacionalização de empresas do Nordeste: a proposição de um framework. Tese (Doutorado) - Programa de Pós-Graduação em Administração - PROPAD. Universidade Federal de Pernambuco -UFPE, Recife, 2009.

.; MORAES, W. A. F.; OLIVEIRA, B. R. B. Internationalization Process of Brazilian Companies.

In: Marin Marinov, Stela Marinova. (Org.). Impacts of Emerging Economies and Firms on International Business. London: Palgrave Macmillan, 2012.

KWEE, Z.; VAN DEN BOSCH, F. A. J.; VOLBERDA, H. W. The Influence of Top Management Team's Corporate Governance Orientation on Strategic Renewal Trajectories - A Longitudinal Analysis of Royal Dutch Shell plc, 1907-2004. Journal of Management Studies, v.48, p.984-1014, July, 2011.

LA PORTA, R.; LOPEZ-DE-SILANES, F.; SHLEIFER, A.; VISHNY, R. Corporate ownership around the world. Journal of Finance, Malden, v.54, n.2, p.471-518, Apr. 1999.

LAM, L. W; WHITE, L. P. An adaptative choice model of the internationalization process. International Journal of Organizational Analysis, v.7, n.2, ApnLp. 105-134, 1999.

LANDSTRÖM, H.; LOHRKE, F. Historical foundations of entrepreneurship research. Great Britain: Edward Elgar Publishing, 2010.

Empreendedorismo internacional: proposição de um framework analítico. Tese (Doutorado) Programa de Pós-Graduação em Administração - PROPAD. Universidade Federal de Pernambuco - UFPE, Recife, 2012.

.; DE MORAES, W. F. A.; SALAZAR, V. S. Expressions of Relationship Networking in International Entrepreneurship. Journal of International Entrepreneurship (Print), v. 13, p. 1-26, 2016.

.; MORAES, W. F. A. Facetas do risco no empreendedorismo internacional. Revista de Administração Contemporânea, v. 18, n. 1, p. 96-117, 2014. 
.; MORAES, W. F. A. As dimensões do Empreendedorismo Internacional: uma proposição de um framework. Revista Ibero-Americana de Estratégia, v. 13, n. 4, p. 91-106, 2014. LIEN, Y-C.; LI, S. Does diversification add firm value in emerging economies? Effect of corporate governance. Journal of Business Research, v.66, p.2425-2430, 2013.

LIN, W.-T. Family ownership and internationalization processes: Internationalization pace, internationalization scope, and internationalization rhythm. European Management Journal, v.30, n.1, p.47-56, feb, 2012.

LINDSTRAND, A; ERIKSSON, K; SHARMA, D. The perceived usefulness of knowledge supplied by foreign client networks. International Business Review, v. 18, n. 1, p. 26-37, 2009.

LU, S.; YAN, H. A comparative study of the measurements of perceived risk among contractors in China. International Journal of Project Management, v.31, n.2, p.307-312, 2013.

LU, Y; ZHOU, L; BRUTON, G.; WEIWEN, L. Capabilities as a mediator linking resources and the international performance of entrepreneurial firms in an emerging economy. Journal of International Business Studies, v. 41, p.119-136, 2010.

LUMPKIN, G. T.; DESS, G. G. Clarifying the entrepreneurial orientation construct and linking it to performance. Academy of Management, v. 21, n. 1, 135-172, p. Jan 1996.

MACHADO, H. P. V.; NASSIF, V. M. J. Réplica - Empreendedores: Reflexões sobre Concepções Históricas e Contemporâneas. Rev. adm. contemp. [online], v.18, n.6, pp. 892-899, 2014.

MAGALHAES, E.; RUPOLO, M.; OLIVEIRA, B. R. Processo de internacionalização de uma empresa do Vale do São Francisco: influência dos agentes externos e das escolhas gerenciais. Internext (São Paulo), v. 9, p. 40-60, 2014.

MAIA, A. B. G. R.; VASCONCELOS, A. C.; DE LUCA, M. M. M. InternexT: Revista Eletrônica de Negócios Internacionais da ESPM, Vol.8(2), pp.40-60, 2013.

MAIS, I.; CARVALHO, L. C.; AMAL, M. Determinantes do desempenho exportador: Abordagem institucional do caso de empresas brasileiras. In: XXXVI Encontro da Associação Nacional de Pós Graduação em Administração, 2012, Rio de Janeiro. In.: Anais... Rio de Janeiro: ANPAD, 2012.

.; CARVAlHO, L. C.; AMAL, M. Redes, inovação e desempenho exportador: uma abordagem institucional. RAC - Revista de Administração Contemporânea (Online), v. 18, p. 551-576, 2014.

MAGACHO, L.; PRESA, M.; CARNEIRO, J. Pipeway Engenharia: o desafio de conquistar o exterior sem arriscar a liderança no mercado doméstico. Tecnologias de Administração e Contabilidade, v. 2, n. 1, p. 89-112, 2012.

MARTINCORENA, I.; SESHASAYEE, A. S. N.; LUSCOMBE, N. M. Evidence of non-random mutation rates suggests an evolutionary risk management strategy. Nature, v.484, n.7396, p.95-104, May, 3, 2012.

MCCARTHY, B.; LEAVY, B. The entrepreneur, risk-perception and change over time: a typology approach. IBAR, v. 20, n. 1, p.126-140, 1999.

MELIN, L. Internationalization as a strategy process. Strategic Management Journal, 13, 1992, p. 99- 118.

MELLO, R. C.; ROCHA, A.; MACULAN, A.-M. A Trajetória Internacional das Pequenas Empresas: É 
Possível Conciliar as Teorias Comportamentais? In: IV Encontro de Estudos em Estratégia, Recife, 2009. Anais... Recife: 3Es, 2009.

MEYER, J. W., ROWAN, B. Institutionalized organizations: formal structures as myth and ceremony. In: Sage, 1992.

;SCOTT, W. R. Organizational environments: ritual and rationality. Updated Edition. London:

MINETTI, R.; MURRO, P.; ZHU, S. C. Family Firms, Corporate Governance and Export. Econômica, Vol.82, pp.1177-1216, 2015.

MINTZBERG, H. Patterns in strategy formation. Management Science, v.24. n.9, p. 934-948, 1978.

.; WATERS, J. A. Of strategies, deliberate and emergent. Strategic Management Journal, v. 6. n.3, p. $257-272,1985$.

MOIZINHO, L. C. S.; BORSATO, R. B.; PEIXOTO, F. M.; PEREIRA, V. S. Governança corporativa e internacionalização: uma análise dos efeitos nas empresas brasileiras. Revista de Ciências da Administração, v. 16, n. 40, p. 104-122, 2014.

MORAES, W. F. A. de, et al. Internacionalização de Grupos Empresariais Transnacionais Brasileiros. Conselho Nacional de Desenvolvimento Científico e Tecnológico - CNPq. Processo 406363/2012-9. Chamada: MCTI/CNPq/MEC/CAPES nº 18/2012 - Edital Ciências Humanas, Sociais e Sociais Aplicadas. 2012.

MOTA, J. C. V.; MACHADO, A. G. C.; MORAES, W. F. A. Condicionantes da internacionalização e desempenho exportador: evidências no setor sucroalcooleiro brasileiro. Custos e @gronegócio Online, v. 10, p. 156-183, 2014.

NARAYANAN, V. K.; FAHEY, L. Análise macroambiental: compreendendo o ambiente fora do setor. In: FAHEY, L.; RANDALL, R. M. MBA: Curso Prático de Estratégia. São Paulo: Campus, p. 214 - 243, 1999.

NASSIF, V. M. J. ; GHOBRIL, A. N. ; SILVA, N. S. . Understanding the entrepreneurial process: a dynamic approach. Brazilian Administration Review, v. 7, p. 213-226, 2010.

NUWAGABA, D.; NTAYI, J. M.; NGOMA, M. Psychic distance, firm size, perceived risk and export market choice of exporting firms in Uganda.(Report). European Scientific Journal, v.9, n.10, p.198- 219, apr.1, 2013.

OLIVEIRA, B. R. B.; COSTA, C. S. R.; SALAZAR, V. S.; KOVACS, E. P.; LEITE, Y. V. P. Strategy Formation Process and Corporate Social Responsibility: the case of CHESF. In: 2012 BALAS Annual Conference Business Association of Latin American Studies, 2012, Rio de Janeiro. Proceedings.. Seattle, WA: BALAS, 2012.

; MORAES, W. F. A.; KOVACS, E. P.; LUCIAN, R. Processos de formação de estratégias Internacionais na Fruticultura Brasileira: uma abordagem integrada. Cadernos EBAPE.BR (FGV. Online), v. VII, p. 2, 2009.

.; SALAZAR, V. S.; CRÊSPO, P. M.; COSTA, C. S. R.; KOVACS, E. P. Estratégia competitiva em MPEs: dimensões do processo e suas associações com as estratégias genéricas e funcionais. Gestão \& Produção (UFSCAR. Impresso), v. 22, p. 119-132, 2015.

OLIVEIRA, J. L.; ALBUQUERQUE, A. L.; PEREIRA, R. D. Governança, Sucessão e Profissionalização em uma Empresa Familiar: (re)arranjando o lugar da família multigeracional. Revista Brasileira de Gestão de Negócios, v.14, n.43, p.176-192, 2012. 
OSLAND, G. E.; TAYLOR, C. R.; ZOU, S. Selecting international modes of entry and expansion. Marketing Intelligence and Planning, v.19, n. 3, 2001.

PARRA VILLANUEVA, L. F. Modelo de desarrollo del Potencial Humano basado en competencias para el desarrollo de la actividad empresarial en la poblacion vulnerable de Santiago de Cali. GESTION DE RECURSOS HUMANOS (Report). Ingenieria Industrial, Sept, v.30, n.3, 2009.

PENG, M. W. The resource-based view and international business. Journal of Management, v. 27, n. 6, p. 803-829, 2001.

; WANG, D. Y. L.; JIANG, Y. An institution-based view of international business strategy: a focus on emerging economies. Journal of International Business Studies, v. 39, p. 920- 936, 2008.

PENROSE, E. T. A economia da diversificação. Revista de Administração de Empresas, Rio de Janeiro, v.4, n¹9, p.7-30, set/out, 1979.

PERKS, K. J. Influences on international market entry method decisions by European entrepreneurs. International Journal of Entrepreneurship, v.13, n. S1, p.1-20, Dec, 2009.

PETERAF, M. A. The cornerstones of competitive advantage: a resource-based view. Strategic Management Journal, v. 14, p. 179-191, 1993.

PETRY, J. F.; BRIZOLLA, M. M. B.; HERCULANO, H. A.; HEIN, N. Influência da internacionalização das empresas brasileiras na criação de valor. Revista Eletrônica de Estratégia \& Negócios, v. 7, n. 1, p. 158-180, 2014.

PETTIGREW, A. M. Context and action in transformation of the firm. Journal of Management Studies, v. 24, n. 6, p. 649-670, Nov. 1987.

PIERCY, N. F. Company internationalisation: active and reactive exporting. European Journal of Marketing, vol. 15 No. 3, pp. 24-40, 1981.

PLATCHEK, R. G. B.; FLORIANI, D. E. O posicionamento internacional das empresas têxteis catarinenses: uma análise qualitativa. Revista Ibero-Americana de Estratégia, v. 12, n. 2, p. 42-68, 2013.

PORTER, M. E. Estratégia competitiva: técnicas para análise de indústrias e da concorrência. Rio de Janeiro: Campus, 1986.

The competitive advantage of nations. New York: Free Press, 1990.

Campus, 1989. Vantagem competitiva: Criando e sustentando um desempenho superior. 16. Ed. Rio de Janeiro:

POSEBELlO, D.; AMAL, M.; HOELTGEBAUM, M. Determinants of international entry mode choice: a case study of a Brazilian multinational. BASE - Revista de Administração e Contabilidade da UNISINOS, v. 10, n. 2, p. 181-194, 2013.

PRAHALAD, C.; HAMEL, G. The core competence of the corporation. Harvard Business Review, v. 68, issue 3, p. 79-91, May/Jun. 1990.

REID, S. D. The Decision-maker and export entry and expansion. Journal of Review, v. 16, n. 1, p. 145-179, 1991. 
RABOCH, H.; AMAL, M. A internacionalização de pequenas e médias empresas: um estudo de caso na região do Vale do Itajaí. In: V EGEPE sobre empreendedorismo e gestão de pequenas empresas, 2008, São Paulo. Caderno de resumos dos trabalhos do V EGEPE sobre empreendedorismo e gestão de pequenas empresas, 2008.

SANTOS, M. C. B. G.; PINHO, M. Estratégias tecnológicas em transformação: um estudo da indústria farmacêutica brasileira. Gestão \& Produção, v.19, n.2, p.405-418, 2012.

SCHWEIZER, R; VAHLNE, J.-E.; JOHANSON, J. Intemationalization as an entrepreneurial process. Journal of International Entrepreneurship, v. 8, n. 4, 2010.

SHANE, S.; VENKATARAMAN, S. The promise of entrepreneurship as a field of research. Academy of Management Review, v.25, n. 1, 217-26, 2000.

SHARPE, W. F. Capital Assent Prices: A Theory of Market Equilibrium under Conditions of Risk. Journal of Finance. Chicago: American Finance Association, v. XIX, n. 3, p. 425-442, Sept. 1964.

SILVA, G.; AMAL, M.; TONTINI, G.; BORGES, G. R. Efeitos das distâncias e recursos da empresa no desempenho exportador de empresas. Internext (São Paulo), v. 10, p. 1-14, 2015.

SILVA, I. M.; CHAGAS, A. C. C. D.; SIQUEIRA, S. V. Características de empreendedorismo internacional no processo de internacionalização: um estudo de caso na empresa Menendez \& Amerino. InternexT - Revista Eletrônica de Negócios Internacionais da ESPM, v. 7, n. 2, p. 107-136, 2012.

SINGLA, C.; GEORGE, R. Internationalization and performance: A contextual analysis of Indian firms. Journal of Business Research, v.66, n.12, p.2500-2506, dec. 2013.

STRANGE, R.; FILATOTCHEV, I.; BUCK, T.; WRIGHT, M. Corporate governance and international business. Management International Review, v.49, p395-407, 2009.

SULLIVAN, D. Measuring the degree of internationalization of a firm. Journal of International Business Studies 25(2): 325-342 1994.

SZCZEPAŃSKI, R.; ŚWIATOWIEC-SZCZEPAŃSKA, J. Risk management system in business relationships — Polish case studies. Industrial Marketing Management, v.41, n.5, p.790-799, 2012.

TORRENS, E. W.; AMAL, M.; TONTINI, G. Determinants of Export Performance of Small and Mediumsized Manufacturing Brazilian Enterprises from the Perspective of Resource-based View and Uppsala Model. Revista Brasileira de Gestão de Negócios (São Paulo. Impresso), v. 16, p. 511, 2014.

VERGA, E.; SILVA, L. F. S. Empreendedorismo: evolução histórica, definições e abordagens. Revista de Empreendedorismo e Gestão de Pequenas Empresas, v. 3, n. 3, p. 3-30, 2014.

VÉRIN, H. Entrepreneurs, enterprise. Histoire d'une idée. Paris: Presses Universitaires de France. 1982.

VERNON, R. International Investments and International Trade in the Product Cycle. Quarterly Journal of Economics. v. 80, 1966.

. The product cycle hypothesis in a new international environment. Oxford Bulletin of Economics and Statistics, Volume 41, Issue 4, pages 255-267, November 1979.

VOLONTÉ, C.; GANTENBEIN, P. Directors' human capital, firm strategy, and firm performance. Journal of Management \& Governance, Vol.20(1), pp.115-145, 2016. 
WELCH, C.; WELCH, L. S. Broadening the concept of international entrepreneurship: internationalization, networks and politics. Journal of International Entrepreneurship, v. 2, n. 3, p. 217-237, Sep. 2004.

WERNERFELT, B. A resource-based view of the firm. Strategic Management Journal, v.5, p.171- 180, 1984.

WILLIAMSON, O. E. The Economic Institutions of Capitalism - Firms, Markets, Relational Contracting. New York: Free Press, 1985.

. The Institutions of Governance. The American Economic Review, v. 88, n. 2, p. 75-79, 1998.

. Transaction cost economics: an introduction. Economics Discussion Papers. Discussion Paper 2007-

3, March 1, 2007. Disponível em: <http://www.economics- ejournal.org/economics/discussionpapers/2007-3>. Acessado em: 2.set.2015

Transaction-Cost Economics: The Governance of Contractual Relations. Journal of Law and Economics, v.22, n. 2, Oct., 1979.

YORKS, L.; NICOLAIDES, A. A Conceptual Model for Developing Mindsets for Strategic Insight Under Conditions of Complexity and High Uncertainty. Human Resource Development Review, v.11, n.2, p.182202, jun. 2012.

ZILLI, J. C.; VIEIRA, A. C. P.; HEINZEN, M. Estratégias e grau de internacionalização de empresas transformadoras de polímeros. Revista de Administração de Roraima - RARR, v.5, n.1, p.23-41, 2015. 